\title{
20
}

\section{Using Formal Methods: if you're so smart how come you ain't rich?}

\author{
E. Brinksma \\ University of Twente \\ P.O. Box 217, 7500 AE Enschede, The Netherlands \\ brinksma@cs.utwente.nl
}

\begin{abstract}
The appreciation of formal methods as useful tools for the design and analysis of communicating systems is subject to considerable variation of opinion. They are seen as a solution by some, and a problem by others. They have been hailed as the answer to the software crisis by some and others have detested them for their obvious inapplicability. Some think their days are yet to come, for others they are part of a now forsaken paradigm. In our presentation we will analyse the reasons for these contradictory views. By reviewing some successful and failed applications of formal methods we will try and find out under what conditions the application of formal methods is profitable.
\end{abstract}

